\title{
Malaria Test Positivity Rates in Health Facilities Supported by the Global Fund in Kinshasa City
}

\author{
Gabriel Kwete Woto Kwete¹, Pélagie Babakazo Diambalula², Joël Nkondi Nkiama², \\ Maurice Bope Bope ${ }^{1}$, Paul Mansiangi Mankadi² \\ ${ }^{1}$ Université Officielle de Mweka, Kasaï, Congo \\ ${ }^{2}$ Ecole de Santé Publique, Faculté de Médecine de l’Université de Kinshasa, Kinshasa, Congo \\ Email: gabrielkwete@gmail.com
}

How to cite this paper: Kwete, G.K.W., Diambalula, P.B., Nkiama, J.N., Bope, M.B. and Mankadi, P.M. (2019) Malaria Test Positivity Rates in Health Facilities Supported by the Global Funds in Kinshasa City. Open Access Library Journal, 6: e5662.

https://doi.org/10.4236/oalib.1105662

Received: August 5, 2019

Accepted: November 25, 2019

Published: November 28, 2019

Copyright (c) 2019 by author(s) and Open Access Library Inc.

This work is licensed under the Creative

Commons Attribution International

License (CC BY 4.0).

http://creativecommons.org/licenses/by/4.0/

\begin{abstract}
The objective of this study was to determine the positivity rates of malaria screening tests for suspected patients and their impact on the supply of management inputs. It was a cross-sectional descriptive study on the structures of care supported by the Global Fund in the city-province of Kinshasa based on a sample which was constituted of 520 patients. The results showed that out of 520 presumed patients, $36.3 \%$ were male and $63.7 \%$ female. Regarding age group, 179 children aged 0 to 5 years or $34.4 \%$ divided into 3 layers according to the prevalence of the disease in the city of Kinshasa. Five hundred and twenty thick drops (TD) were performed including 139 positive $(26.7 \%)$ and 381 negative ones (73.3\%). In children, 54 thick drops (TD) out of 179 (30.1\%) were positive. The highest positivity rate was observed in stratum 3 (30.2\%). From these results, it should be said that excessively high positivity rates lead to an oversupply of input structures resulting in excess stocks and peremptions. The exact positivity rate should be known throughout the Democratic Republic of Congo (DRC) to better plan and monitor malaria control activities.
\end{abstract}

\section{Subject Areas \\ Epidemiology, Infectious Diseases}

\section{Keywords}

Positivity, Malaria, Test, Suspected Cases, Kinshasa City

\section{Introduction}

Malaria is one of the most common parasitic diseases in the world. It is caused 
by plasmodium-like parasites transmitted to humans by bites of infected female Anopheles mosquitoes [1]. Four main species are involved: Plasmodium falciparum, P. ovale, P. vivax and P. malariae [2]. Globally, it is a public health priority as it is one of the leading infectious causes of mortality in endemic areas. Outside these areas, we are talking about import malaria. It is a diagnostic and therapeutic emergency especially when it comes to its severe form [3].

The disease continues to have devastating consequences on health, which weighs heavily on the health of the world. That is why it was one of the objectives of MDG 6, to "Reduce the burden of malaria by 2015". In 2015, 214 million cases of malaria and 438,000 deaths were reported. More than two-thirds or 70\% of these deaths occurred in children under 5 years of age and 95 countries and territories experienced continued transmission of the disease [4].

Malaria remains a major public health problem in the Democratic Republic of Congo. It still remains at the top of the causes of consultation, hospitalization and death. In the analysis of the situation contained in the National Health Development Plan (NHDP) 2011-2015, malaria is responsible, with acute respiratory infections, diarrhea and neonatal diseases, for $77 \%$ of deaths in children under the age of 5 years in the DRC. However, remarkable progress has been made between 2010 and 2014 in reducing malaria morbidity (10\%) and infant mortality has decreased by $37 \%$, from $92 \%$ in 2010 to $58 \%$ in 2014, of which $33 \%$ relate to the prevention of malaria [5].

To combat this endemic in the DRC, the Ministry of Public Health, under the National Malaria Control Program (NMCP), has officially adopted the Roll Back Malaria initiative since 2001.

With regard to diagnosis, since the beginning of 2010, the WHO recommends, before treatment, a rapid biological confirmation by microscopic thick blood drop examination (TD) or Rapid diagnostic test (RDT) to all malaria suspected patients [5].

In tropical Africa, the diagnosis of malaria is based on clinical evidence, including fever in the absence of biological confirmation (RDT or TD). An appropriate treatment is unfairly put in place. This situation is the same in the DRC in the majority of cases.

However, some studies have shown that the presumption of access to malaria was only confirmed in less than $50 \%$ of cases. This rate can be even lower if the diagnosis is made by paramedical personnel.

Parasitological examination is the only way to diagnose malaria in a febrile patient safely. Treating a patient on the basis of such an examination offers many advantages related to the presumptive treatment of all febrile attacks. This argument is also reinforced by the fact that the positivity rate of malaria screening tests in febrile patients is far less than $100 \%$, the latter being different from one country to another. According to the WHO, the positivity rate of RDTs for sub-Saharan Africa was 44\% in 2014 and 52\% in 2015 [6]. On the other hand, health facilities in the DRC report higher rates than the WHO average of $67 \%$ for 2013 according to the NMCP [4]. 
The objective of this study is to determine the positivity rates of malaria tests in suspected cases in health care structures of health districts benefiting from the intervention of the Global Fund in the city of Kinshasa and assess the impact on the quality of malaria supply structures in these facilities.

\section{Materials and Methods}

\subsection{Site Description and Period of Study}

This study was conducted from December 1, 2016 to February 20, 2017 in health care facilities in five health districts (HD) in Kinshasa as part of a Global Fund intervention. These health structures are in the 3 layers of the geographical distribution of malaria discovered by Ferrari et al. [7], as shown in Figure 1.

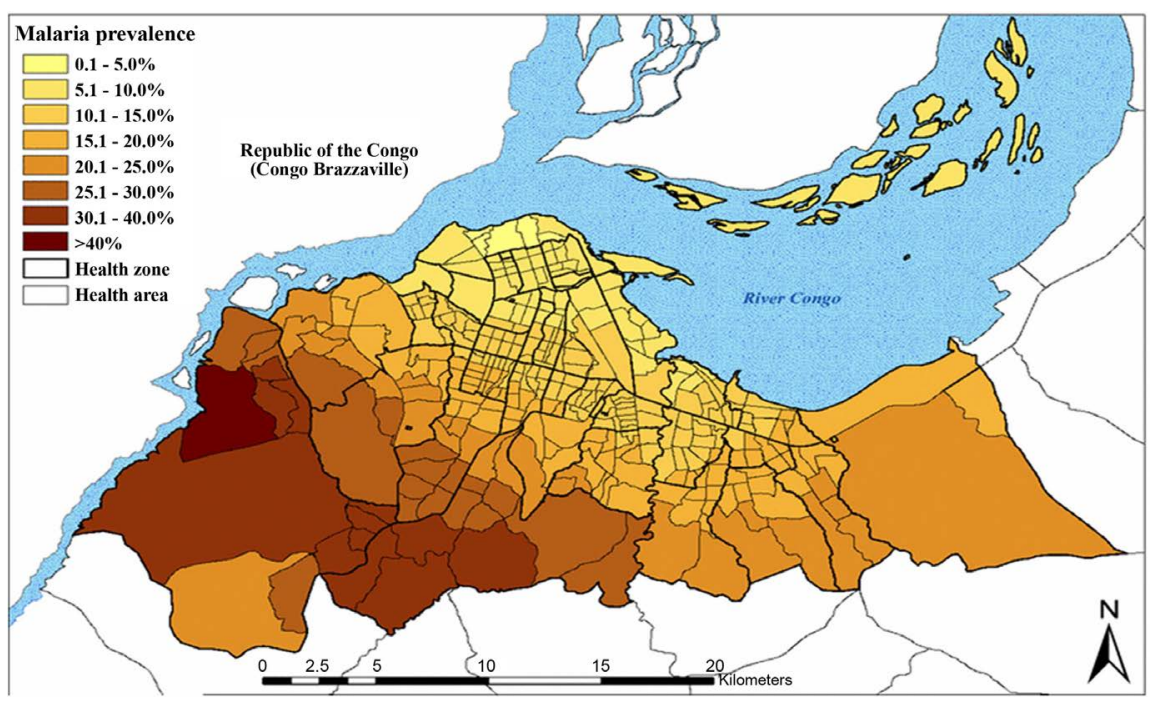

Figure 1. Geographic distribution by Health Square (HS) of Falciprum Plasmodium (FP) malaria in children aged 6 to 59 months in the city of Kinshasa (Source: Ferrari et al., Malaria Map Risk/DRC).

\subsection{Study Population and Statistical Units}

The study concerned all malaria suspected patients who were consulted in the selected health facilities of the 7 HDs of the city-Kinshasa Province. Therefore, are concerned the patients who were consulted for fever or fever history 48 hours before the consultation in the various health structures selected during the period from December 1, 2016 to February 20, 2017 which were considered as the main statistical units of our study.

\subsection{Samples Choice}

\subsubsection{Determination of the Patient Sample Size}

The calculation of the sample size of patients was based on the following formula:

$$
n \geq(g) *(Z / d)^{2} * p *(1-p)
$$

In which the different symbols represent respectively: 
$g$. Factor that accounts for cluster effect $(g=2)$, HDs and health structures.

$Z$ : Coefficient of Confidence (for a 95\% confidence level, $Z=1.96$ )

$d$ : Risk of error (for a margin of error of $5.0 \%, d=0.05$ )

$p$ : The prevalence of malaria confirmed by the TD for the city of Kinshasa, reported in the DHS 2013-2014, is $18.1 \%$.

$$
n=g * Z^{2} \frac{p *(1-p)}{d^{2}} \text { or } n=2 *(1.96)^{2} \frac{(0.18) *(1-0.18)}{(0.05)^{2}}=454
$$

To account for possible non-responses, $15 \%$ of the minimum sample size calculated (68 suspect cases) was added. This brings this size up to 522 patients. 520 patients were selected.

\subsubsection{Sources and Techniques of Data Collection}

The data were collected from the cards of patients admitted for fever or fever history 48 hours before the consultation during the period of our study or in any patient whose diagnosis of malaria was made by the clinician, the blood sample was done by a laboratory technician, the slides were colored and read in the laboratory of the Kinshasa School of Public Health by an experienced technician, the reason why no quality control was required.

\subsubsection{Sampling Technique}

We used probabilistic two-stage sampling: Health Districts and Health Facilities.

\subsubsection{Selection of Health Districts}

Referring to the stratification of Giovanfrancesco et al. (2016) on the mapping of the risk of malaria in Kinshasa [7], the HDs of Kinshasa were grouped into 3 strata:

1) Low-risk HDs with a prevalence of malaria less than $5 \%$;

2) Intermediate risk HDs with a prevalence of malaria between 5 and 30\%;

3) High risk HDs with malaria prevalence greater than $30 \%$.

Taking into account the weight of each stratum, the following distribution was selected: 2 out of $6 \mathrm{HDs}$ of stratum 1.4 out of $36 \mathrm{HDs}$ of stratum 2 .

The Biyela HD being the only one constituting the stratum 3, was automatically selected.

For the first 2 strata, the HD were selected in a simple random manner. The different selected HDs are presented in Table 1.

This table gives us the selected HDs for our study according to the geographical distribution of malaria in the city-province of Kinshasa concerning the prevalence of the disease.

\section{Results and Discussion}

\subsection{Characteristics and Distribution of Respondents}

In that study, 520 malaria suspects were selected from 8 facilities in the Biyela, Gombe, Kingabwa, Lingwala, Lemba, N'djili and Police Health Districts. Table 2 summarizes the distribution of respondents by age, sex, HD, and stratum 
Table 1. Health districts selected by Strata.

\begin{tabular}{ccc}
\hline STRATE 1 & STRATE 2 & STRATE 3 \\
\hline & GOMBE & \\
Kingabwa & LEMBA & BIYELA \\
& N'DJILI & \\
Lingwala & POLICE & \\
\hline
\end{tabular}

Table 2. Sociodemographic characteristics and distribution of respondents.

\begin{tabular}{|c|c|c|c|}
\hline Characteristics & Division & $\mathrm{n}=520$ & $\%$ \\
\hline \multirow[t]{6}{*}{ Health District } & Gombe & 26 & 5.0 \\
\hline & Biyela & 173 & 33.3 \\
\hline & Kingabwa & 148 & 28.5 \\
\hline & Lingwala & 86 & 16.5 \\
\hline & Lemba & 86 & 16.5 \\
\hline & Police & 32 & 6.2 \\
\hline \multirow[t]{3}{*}{ Stratum } & Strate 1 & 173 & 33.3 \\
\hline & Strate 2 & 174 & 33.4 \\
\hline & Strate 3 & 173 & 33.3 \\
\hline \multirow[t]{2}{*}{ Sex } & Male & 189 & 36.3 \\
\hline & Female & 331 & 63.7 \\
\hline \multirow[t]{4}{*}{ Age in slices } & $0-5$ years & 179 & 34.4 \\
\hline & 6 to 10 years & 138 & 26.5 \\
\hline & $11-16$ years & 101 & 19.4 \\
\hline & Over 16 years old & 102 & 19.6 \\
\hline
\end{tabular}

of belonging. Female patients were the majority (63.7\%) compared to men (36.3\%) and the age group from 0 to 5 years were $34.4 \%$ of respondents and $20 \%$ of these were aged just over 16 years; the median age was 23 , the youngest patient was less than a year old and the oldest was 91 and the proportion of respondents per stratum was $33.3 ; 33.4$ and $33.3 \%$ for strata 1,2 and 3. Of all the Health Districts, it is Biyela who had a large proportion of patients because they belong alone to the 3 rd stratum (33.3\%).

\subsection{The Different Biological Tests Carried Out}

As for the various biological tests carried out as well as their rate of implementation, it is to be demonstrated in this study that $100.0 \%$ of the patients have made the biological test of the thick drop. With regard to RDT, 70.4\% of patients underwent this test because there was a problem of availability in some structures with an achievement rate of $78.2 \%$ in stratum 2 (174 patients) compared to other strata. This SD Bioline Malaria AG pf/Pan RDT test was made available in the 
structures by the SANRU/FM Project, but many of the cases of stock-outs were encountered in some health facilities, which did not allow us to do so to examine all the patients.

\subsection{The Results of the Biological Tests Carried Out}

\subsubsection{Comparison between TD and RDT}

Although carried out at different proportions, it was important during this study to compare the results of these 2 diagnostic biological tests submitted to the suspect patient. Table 3 shows that in all of the 520 patients examined, $26.7 \%$ with a confidence interval of [22.9 - 31.0] was diagnosed positive for TD compared with positive RDT results is $18.9 \%$ with a confidence interval: CI [5.3 - 23.0].

\subsubsection{Thick Drop (TD) Results by Sex}

The results of this study inform us that out of a total of 520 patients, we counted 189 men and 331 women. To show trends by sex, Figure 2 shows that there is not a significant difference between the results of the thick positive blood test found in male patients (28.6\%) than found in female patients (25.7\%).

\subsubsection{TD Results by Age Group}

The prevalence and severity of malaria are not the same in children and adults, which led us to release the results by age group. The figure above shows that it is in the 0 to 5 age group that the positivity rate (30.2\%) is high compared to other age groups (Figure 3 ).

\subsubsection{Results of TD in Different Strata}

To compare this study with those carried out on the prevalence of malaria in the city of Kinshasa and the risk mapping of this disease, it is necessary to group the results by stratum.

As a result, Table 4 shows that it is Stratum 3 that a large number of patients were diagnosed with the thick positive drop (30.1\%).

\subsubsection{Result of the RDT in Relation to Age}

Although performed at different proportions, it is imperative in this study to compare the results of these two diagnostic tests submitted to the patients. Figure 4 gives the results of RDT performed in different structures. It is the age group of 6 to 10 years that the positivity rate is the highest (23.9\%).

Table 3. Results of the biological tests carried out.

\begin{tabular}{ccc}
\hline Thick drop & $\mathbf{n}=\mathbf{5 2 0}$ & $\%(\mathrm{CI})$ \\
\hline Positive & 139 & $26.7(22.9,31.0)$ \\
Negative & 381 & $73.3(69.0,77.1)$ \\
Rapid diagnostic test (RDT) & $\mathbf{n}=\mathbf{3 6 6}$ & $18.9(15.3 ; 23.0)$ \\
Positive & 69 & $81.1(77.0,84.7)$ \\
Negative & 297 &
\end{tabular}




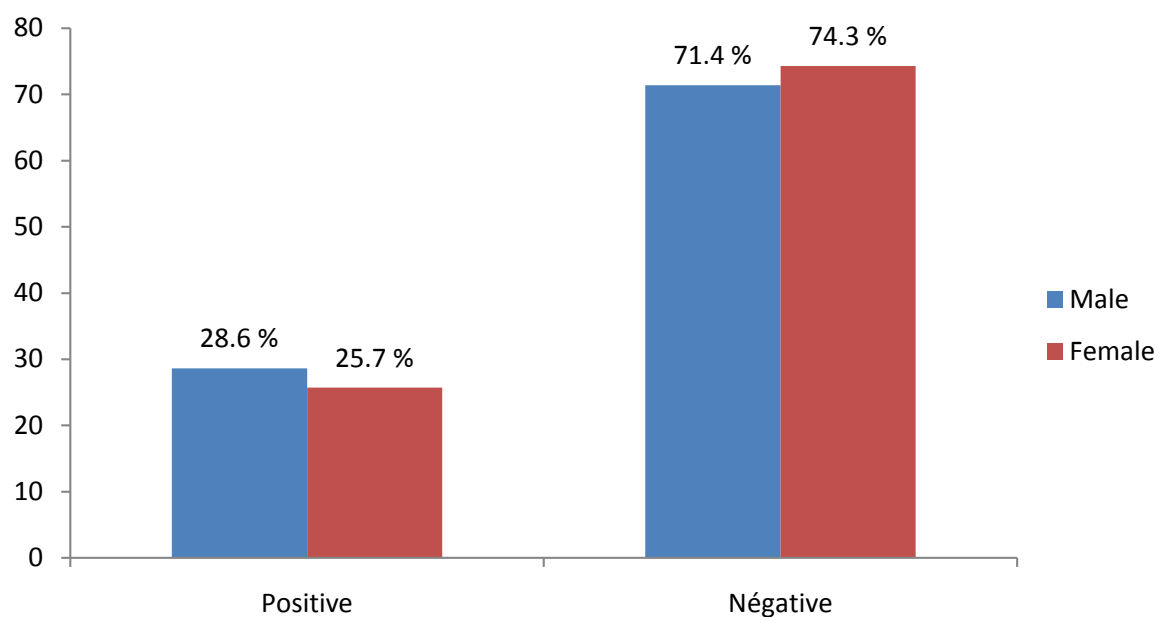

Figure 2. TD results by sex.

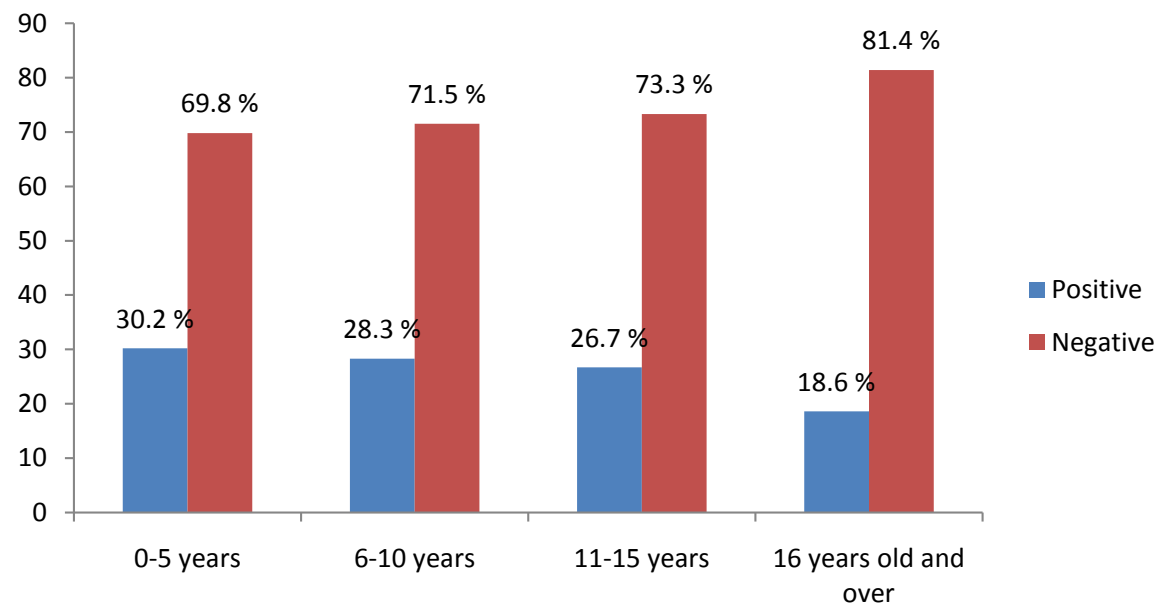

Figure 3. TD score by age group.

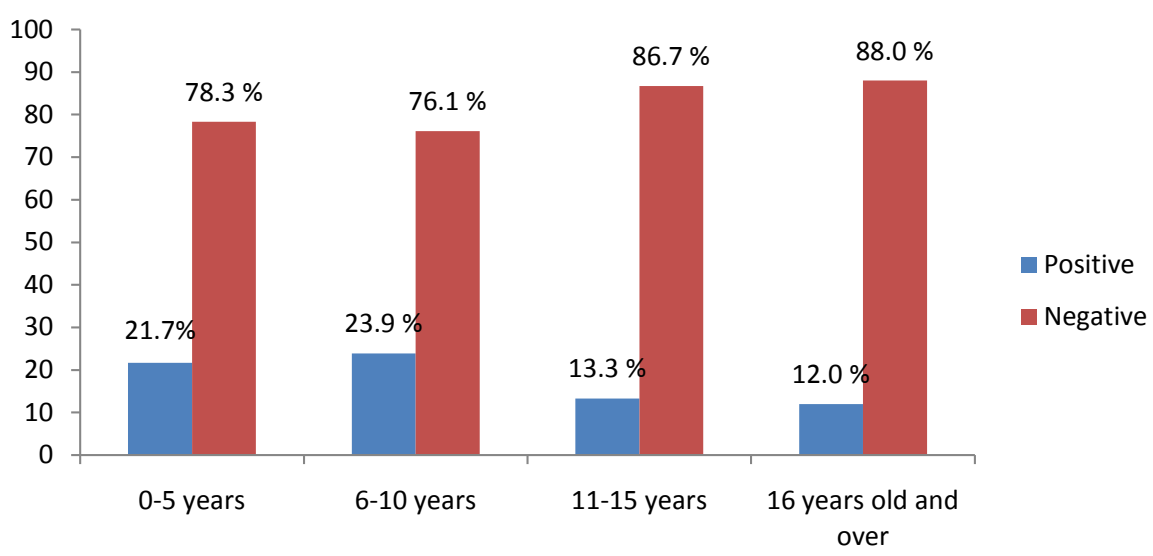

Figure 4. RDT score by age group.

\subsubsection{The Validity of the RDT}

Since the positivity rate of RDT was much lower than that of TD, we calculated from the data in Table 5 the sensitivity, specificity and positive and negative predictive values to evaluate the validity of this test. The results found showed 
Table 4. Results of TD by stratum.

\begin{tabular}{cccc}
\hline Stratum & Effective & Positive & Negative \\
\hline Strate 1 & 173 & $41(23.7 \%)$ & $132(76.5 \%)$ \\
Strate 2 & 174 & $46(26.4 \%)$ & $128(73.6 \%)$ \\
Strate 3 & 173 & $52(30.1 \%)$ & $121(69.9 \%)$ \\
Total & $\mathbf{5 2 0}$ & $\mathbf{1 3 9 ( 2 6 . 7 \% )}$ & $\mathbf{3 8 1 ( 7 3 . 3 \% )}$ \\
\hline
\end{tabular}

Table 5. RDT validity with respect to TD.

\begin{tabular}{ccccc}
\hline & & \multicolumn{2}{c}{ RDT } & \multirow{2}{*}{ TOTAL } \\
\hline \multirow{3}{*}{ TD } & POSITIVE & NEGATIVE & \\
\hline & NEGATIVE & 20 & 66 & 115 \\
& TOTAL & 69 & 231 & 251 \\
& & 69 & 297 & 366 \\
\hline
\end{tabular}

that the sensitivity of the RDT is $71.0 \%$, the specificity of $77.8 \%$, the positive predictive value of $42.6 \%$ and the negative predictive value of $92.0 \%$.

\section{Discussion}

The objective of this study was to determine the positivity rates of the malaria tests in 103 structures of the city-province of Kinshasa. Blood samples were collected from laboratories in these different facilities and patient information was collected through interrogation and through medical charts. The slides were read at the laboratory of the School of Public Health in Kinshasa.

\subsection{The Different Biological Tests Carried Out}

Thick drop was performed on all suspected patients in the targeted health facilities, whereas RDT was only $70.4 \%$ achieved due to lack of availability of this input in some health facilities. This result is higher than that reported in the 2013-2014 DHS (19\%) [1] and that reported by the NMCP in its 2013 annual report (53.6\%) [4]. The 2016 WHO World Malaria Report mentions a $76 \%$ rate of achievement of TDR in sub-Saharan African countries [6]. This means that almost $30 \%$ of malaria diagnoses in health facilities are presumably posed.

\subsection{The Results of Biological Tests Carried Out to Diagnose Patients}

This part concerns the discussion of the results of TD and RDT according to the age, gender and strata of belonging of the patients. For RDT, its validity was assessed against the heavy drop.

\subsubsection{The Positivity Rate of TD}

The positivity rate of TD of $26.7 \%$ found in this study is lower than those reported in most studies and some reports in sub-Saharan Africa and the DRC, 
these include SANRU which reported 71\% in 2015 [8], WHO/Burundi in its 2014 annual report mentioned a TD positivity rate of 53.6\% [9], the $\mathrm{NMCP} / \mathrm{DRC}$ in its 2013 annual activity report (63.2\%) [4], and in Mozambique by Mabunda et al. who found 37.8\% [10].

This result is higher than that found by SA Haidaro et al. In Mali, this found $12.8 \%$ among suspected patients hospitalized in internal medicine at Point G Hospital in Bamako [11].

In children, the positivity rate of $30.2 \%$ found in this study is higher than that reported by DRC/DHS 2013-2014, 23\% [1] and those reported by A. Munier et al. In Senegal and F, Tietche et al. in Cameroon respectively $54.4 \%$ and $43.25 \%$ [12] [13]. With regard to the results of TD by stratum, our study confirms the stratification of Ferrari G et al. with regard to the result of strata 2 and 3 which come first in terms of positivity (26.4\% and 30\%).

\subsubsection{The Positivity Rate and the Validity of the RDT}

The positivity rate of RDT found in our study was $18.9 \%$. This result is lower than that found in most studies conducted in the world and DRC. DRC/DHS 2013-2014, found in children a rate of 31\% [1] and yet WHO in its report on malaria in the world for the years 2014 and 2015 had reported rates of $44 \%$ and $52 \%$ respectively [6].

In its annual report on activities in 2013, the NMCP reported that $41,037,745$ out of 6,096,993 completed RDTs were positive, a positivity rate of $67.3 \%$ [12]. Regarding the validity of this test with respect to TD, we noted low values of sensitivity, specificity and positive predictive value (respectively $71 \%, 77.8 \%$ and $42.6 \%$ ) with a positivity rate of RDT. of $31 \%$ in children, this positive predictive value reflects a low extrinsic validity of this test.

\section{Conclusions}

The evaluation of the positivity rate of malaria tests in suspected cases in health zones supported by the Global Fund in the city-province of Kinshasa showed that the thick-blood drop (TD) was performed only in patients making severe malaria even in settings where RDT was not available. The low validity of the $\mathrm{RDT}$, with a $71 \%$ of sensitivity, $77.8 \%$ of specitivity and a predictive value of $42.6 \%$, suggests the poor quality of this test especially when the diagnosis is negative in the case where the patient has high fevers.

In the DRC, the NMCP changed its malaria management policy by adopting artemisinin-based combination therapy as a first-line treatment and RDT as a malaria confirmatory test in health centers. This action should play a key role in improving the quality of malaria diagnosis and in providing reliable data on malaria morbidity essential for better planning and evaluation of control measures. In addition, the more specific identification of incorrect practices related to malaria diagnosis should enable health authorities and health system partners working to improve patient management to better target their interventions. 


\section{Conflicts of Interest}

The authors declare no conflicts of interest regarding the publication of this paper.

\section{References}

[1] DRC Ministry of Planning and Follow-Up of the Implementation of the Revolution of Modernity: "Second Demographic and Health Survey"(DHS II 2013-2014), Kinshasa, 2014, 189-210.

[2] Khovidhunkit, W., Kim, M.S., Memon, R.A., Shigenaga, J.K., Moser, A.H., et al. (2004) Effects of Infection and Inflammation on Lipid and Lipoprotein Metabolism: Mechanisms and Consequences to the Host. The Journal of Lipid Research, 45, 1169-1196. https://doi.org/10.1194/jlr.R300019-JLR200

[3] Laurent, V., Hilly, J., Bedel, J., Planquette, B., Legriel, S., et al. (2014) Paludisme grave d'importation de L'adulte. Journal Européen des Urgences et de reanimation, 26, 97-104. https://doi.org/10.1016/j.jeurea.2014.05.007

[4] Ministry of Public Health/DRC (2014) National Malaria Control Program, PNLP, 2013 Activity Report. Kinshasa, 20-21.

[5] Ministry of Public Health/DRC (2016) National Health Development Plan (PNDS 2016-2020). Kinshasa, 1-84.

[6] WHO. World Malaria Report. Geneva: World Health Organization. https://www.who.int/malaria/publications/world-malaria-report-2016/report/en/

[7] Ferrari, G., Ntuku, H.M., Schmidlin, S., Diboulou, E., Tshefu, A.K. and Lenger, C. (2016) A Malaria Risk Map of Kinshasa/Democratic Republic of Congo. Malaria Journal, 15, 27. https://doi.org/10.1186/s12936-015-1074-8

[8] SANRU/GlobalFund (2016) Malaria Activity Report 2015. Kinshasa.

[9] WHO. Burundi Malaria report 2014, Bujumbura, Juin 2015, p 14.

[10] Mabunda, S., Aponte, J.J., Tiago, A., et al. (2009) A Country-Wide Malaria Survey in Mozambique. II. Malaria Attributable Proportion of Fever and Establishment of Malaria Case Definition in Children Across Different Epidemiological Settings. Malar J, 8,74.

[11] Haidaro, S.A., Doumbo, O., Traore, H.A., Koita, O., Dembélé, M., Dolo, A., et al. (1991) La place du paludisme dans les syndromes fébriles en Médecine Interne à l'Hopial du point G à Bamako: Résultats d'une année d'étude systématique. Médecine d Afrique Noire, 38, 110-117.

[12] Munier, A., Diallo, A., Sokhna, C., Senghor, P. and Chippaux, J.P. (2009) Evaluation of the Reliability of the Clinical Diagnosis of Malaria among Children Consulting in Three Peripheral Health Posts, in the Rural Area of Niakhar in Senegal. Bulletin de la Société de Pathologie Exotique, 120, 113-118.

[13] Tietche, F., Teguia, S., Tetanye, E., Louis, F.J., Mbonda, E. and Epee, M.F. (1996) Presumptive Diagnosis of Malaria Access and Positivity of the Thick Blood Drop in Children Aged 0-5 Years in Yaundé (Cameroon). Medicine of Black Africa, 43, 318-321. 